\title{
A METHOD FOR GOLD COATING EXPERIMENTAL DETECTOR BEAMPIPES*
}

\section{$\underline{\text { S. Henderson }}^{\dagger}$, S. Roberts, Cornell University, Ithaca, NY}

\begin{abstract}
A thin metallic coating is required on the inside of central detector beryllium beampipes at high-current $e^{+} e^{-}$ colliders in order to suppress detector backgrounds that arise from scattered synchrotron radiation. As part of two CESR/CLEO upgrades, three beryllium beampipes have been coated with gold using a magnetron sputtering technique. The apparatus will be described as well as the factors that influence the adhesion and uniformity of the resulting films. Finally, we discuss the characterization methods of the resulting films.
\end{abstract}

\section{INTRODUCTION}

High luminosity $e^{+} e^{-}$colliders require that unprecedented beam currents be brought into collision, while the high-energy experimental requirements demand that beaminduced detector backgrounds be minimized. Such beamgenerated detector backgrounds arise from interaction of beam particles with residual gas in the vacuum chamber (beam-gas interactions) and from synchrotron radiation (SR) generated in nearby magnetic elements which scatters into the detector. Careful interaction region design [1] provides shielding of the detector for lost beam particles and shielding of the central detector beampipe from synchrotron radiation by careful placement of apertures. Even with apertures placed to eliminate direct SR that would otherwise strike the beampipe directly, the scattered radiation from these apertures is great enough that further suppression is required. A common method for further suppressing the scattered SR flux which is in use at the Cornell Electron Storage Ring (CESR), PEP-II, KEK-B and elsewhere is to apply a thin metallic coating to the inner surface of the collider detector beampipe (which is generally made of beryllium). Since the scattered SR flux strikes the beampipe at very shallow angles, very thin metallic layers provide adequate suppression of the scattered SR flux entering the detector.

As part of two recent upgrades to CESR and the CLEO detector [2,3], we have developed a technique for coating the inside of small-radius $(r \simeq 2 \mathrm{~cm}$ ) beryllium beampipes with gold. For the CESR Phase II upgrade, a $10 \mu \mathrm{m}$ gold coating was required, and for the upcoming CESR Phase III upgrade, a $5 \mu \mathrm{m}$ layer is required. Both CLEO detector beampipes are double-walled, actively cooled $\mathrm{Be}$ beampipes manufactured by Brush Wellman-Electrofusion $[4,3]$. The inner radius and length of the as-coated beampipe assemblies were $1.9 \mathrm{~cm}, 43 \mathrm{~cm}$ (Phase II) and

\footnotetext{
${ }^{*}$ Work supported by the National Science Foundation.

† Email: sdh9@ cornell.edu
}

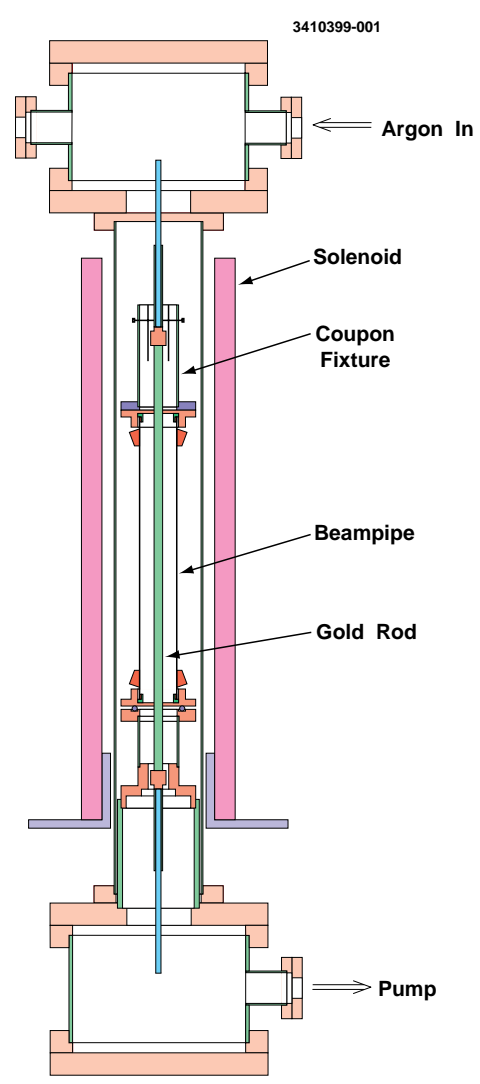

Figure 1: Beampipe coating apparatus

$2.1 \mathrm{~cm}, 36.5 \mathrm{~cm}$ (Phase III), respectively. We describe the deposition technique, the coating "recipe" and the characterization of the resulting thin films.

\section{COATING METHOD AND APPARATUS}

We use a cylindrical magnetron sputtering technique [5] for application of the gold coating. Due to the small radius of the beampipes, DC-diode sputtering without magnetic field was not practicable, since high sputtering gas pressures would be required ( $\sim 300$ mTorr) to sustain a discharge. Sputtering at such high pressures results in very slow deposition rates and in films with undesirable surface structure. As a result, we sputtered in the presence of an axial magnetic field, which allows a discharge to be maintained at much smaller sputtering gas pressures; acceptable deposition rates result because of the enhanced ionization by secondary electrons as they travel in cycloidal, rather than straight-line, paths.

The coating apparatus which was recently used for the CESR Phase III/CLEO-III Be beampipe is shown in Figure 1. The Be beampipe is mounted to a stand which is elec- 
trically isolated from the chamber by ceramic standoffs. Atop the beampipe is mounted a small chamber to hold witness coupons which are coated along with the beampipe for quality control purposes. A water-cooled gold rod is mounted axially in the beampipe. This rod consists of a $1 \mathrm{~mm}$ thick layer of gold clad on $1 / 4$ inch stainless steel tubing. Water cooling of the cathode is essential as very high power densities are reached at the surface due to ionbombardment.

The beampipe and gold rod assembly sits inside an ultrahigh vacuum system with large chambers on top and bottom for instrumentation, feedthroughs, and access. Highvoltage water feedthroughs provide voltage and cooling water to the gold rod. The axial magnetic field is provided by a solenoid placed external to the vacuum system. The system is pumped with a $500 \mathrm{l} / \mathrm{s}$ turbo pump. Chamber pressure is monitored with a Granville-Phillips StabilIon $^{T M}$ hot filament gauge. Argon (UHP 99.999\%) is introduced at the top of the chamber and is pumped at the bottom of the chamber, ensuring flow through the pipe. A commercial magnetron gun power supply is used for sputtering. An electrical connection to the beampipe is brought via a feedthrough outside the vacuum chamber. For backsputtering of the beampipe (see below), high-voltage leads on the beampipe and gold rod are reversed. A thermocouple monitors the temperature of the witness coupon chamber during processing.

\section{COATING RECIPE AND SPUTTERING PARAMETERS}

The sputtering conditions and coating recipe were chosen to maximize the adhesion of the deposited film and to assure its longitudinal uniformity. Each will be discussed in turn.

\subsection{Factors affecting adhesion}

As is the case with any thin-film deposition, cleanliness of the substrate, cathode and vacuum system is the single most important factor in achieving an adherent film. Standard ultra-high vacuum handling techniques were followed for all components entering the vacuum chamber. All fixtures were acid etched prior to initial introduction into the vacuum system. Subsequent handling of fixtures was followed by scrubbing in soap and water, ultrasonic cleaning in Liquinox, and rinsing in isopropanol.

We found that cleanliness alone was not sufficient for acceptable adhesion of gold on beryllium or stainless steel. Three additional steps were required. First, prior to deposition, the Be beampipe was backsputtered. That is, the beampipe itself was sputtered in order to remove surface contaminants. In addition to cleaning the surface, backsputtering may also help to remove the $\mathrm{BeO}$ layer. Secondly, we found that a thin layer of $\mathrm{Cr}(\sim 150 \mathrm{~nm})$ deposited on the Be substrate prior to gold deposition improved the adhesion. This technique of using a $\mathrm{Cr}$ interlayer is com- mon in the semiconductor industry [6] and is thought to improve adhesion by bonding to the oxide layer [7]. Finally, after gold deposition the beampipe is vacuum baked at $300^{\circ} \mathrm{C}$ for 1 hour. This relatively low temperature bake is thought to promote diffusion at the Au-Be interface and thus improve adhesion $[8,9,7]$.

In addition we found that proper Ar flow was essential for achieving adherent films. Argon was introduced at one end of the sytem and made to flow preferentially through the beampipe in order to supply fresh Ar to the discharge. Argon was pumped through the other end of the chamber always at the maximum possible throughput allowed by the turbo pumping system.

\subsection{Factors affecting uniformity}

We found three factors which strongly influenced the resulting gold thickness uniformity: 1) solenoid length, 2) magnetron operating point, and 3) cathode uniformity. The reduced magnetic field at the end of the solenoid produces a weaker discharge at the ends. We used a solenoid $66 \mathrm{~cm}$ in length whereas the beampipes were $\sim 40 \mathrm{~cm}$ in length. Care was taken to center the beampipe in the middle of the solenoid.

The operating region of the apparatus in magnetron mode is shown in Figure 2. For a given Argon gas pressure there is a minimum magnetic field required to sustain a plasma discharge. This minimum field is that which provides sufficient ionization by secondary electrons to just make up for losses, and is approximately given by the field for which secondary electron trajectories just miss the beampipe wall, thus making full cycloidal orbits in the system. In addition, there is a maximum magnetic field above which the discharge is extinguished. This is approximately the magnetic field for which the secondary electron pathlength is shorter than the mean-free-path and hence secondary electrons are recaptured at the cathode surface. We found that the choice of operating point affects the resulting uniformity.

We chose to operate the magnetron at $P_{A r}=4 \mathrm{mTorr}$ and $B=250 \mathrm{G}$. This is sufficiently well removed from the extinguish lines of Figure 2 so that we acheive sputtering along the whole length of the rod despite the small drop in magnetic field at the ends due to finite solenoid length. For gold sputtering, we typically operated at $150 \mathrm{~W}(750 \mathrm{~V}, 0.2$ A), which provided a deposition rate of $\sim 3 \mu \mathrm{m} / \mathrm{hr}$.

Finally, we found that longitudinal nonuniformity of the cathode can deform the plasma discharge and produce longitudinally non-uniform coatings. We found that the plasma discharge was enhanced in regions where the cathode was of smaller radius. Nonuniformity in cathode radius of only $\sim 5 \%$ is significant and can produce non-uniform deposition profiles (see below).

\section{COATING PROCEDURE}

The step-by-step coating procedure is as follows. The magnetron supply power, magnetic field, and flowing Ar gas 


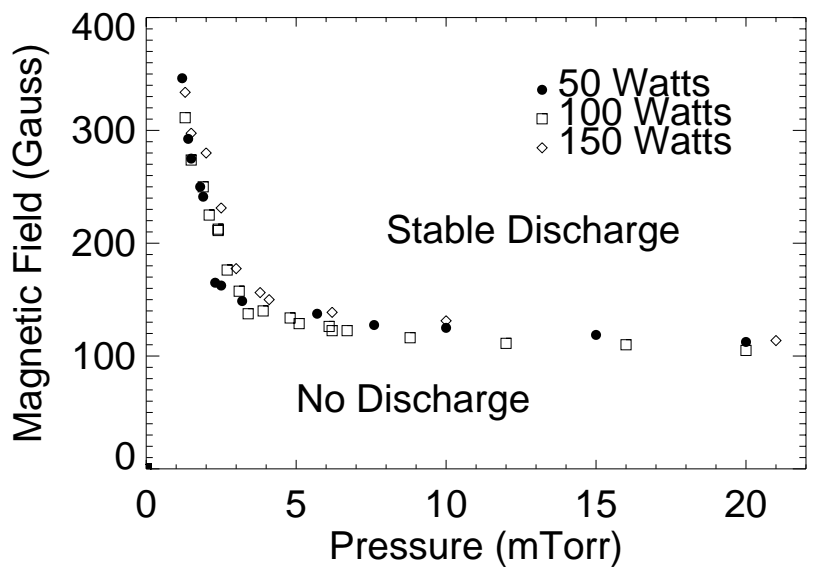

Figure 2: Magnetron operating region. Above the points a discharge is self-sustained. Below the points the discharge extinguishes.

pressure are noted.

1. Clean all fixture parts by scrubbing with abrasive pad and soap. Place in ultrasonic bath with Liquinox. Rinse in isopropanol and blow dry with $\mathrm{N}_{2}$.

2. Assemble fixtures, Be beampipe and $\mathrm{Cr}$ rod. Place in vacuum system. Pump down.

3. Backsputter Be beampipe: $100 \mathrm{~W}(250 \mathrm{~V})$ for $15 \mathrm{~min}-$ utes, 275 mTorr Ar, $B=0$.

4. Apply $150 \mathrm{~nm} \mathrm{Cr}: 100 \mathrm{~W}(600 \mathrm{~V})$ for 9 minutes, 4.0 mTorr Ar, $B=250 \mathrm{G}$.

5. Open vacuum system when cool, replace $\mathrm{Cr}$ with $\mathrm{Au}$ rod, reinstall in vacuum system and pump down.

6. Backsputter Be beampipe: $100 \mathrm{~W}(250 \mathrm{~V})$ for $15 \mathrm{~min}$ utes, 275 mTorr Ar, $B=0$.

7. Apply $5 \mu \mathrm{m} \mathrm{Au}: 150 \mathrm{~W}(750 \mathrm{~V})$ for 90 minutes, 4.0 mTorr Ar, $B=250 \mathrm{G}$.

8. Remove assembly from vacuum system when cool.

9. Bake in vacuum furnace at $300^{\circ} \mathrm{C}$ for 1 hour.

\section{RESULTS}

Throughout the development of the coating procedure, coupons of Be and Stainless Steel were coated for various sputtering conditions. Two adhesion tests were found to be the most useful. The first is the simple "scotch-tape test" in which adhesive tape is attached to the film and pulled quickly in an attempt to remove the film. The second more demanding test requires scribing a grid of finely spaced cuts through the film down to the substrate (typically a $4 \times 4$ grid with cuts spaced $\sim 1 \mathrm{~mm}$ apart). This portion of the coupon was then subjected to the tape test. Generally, if a

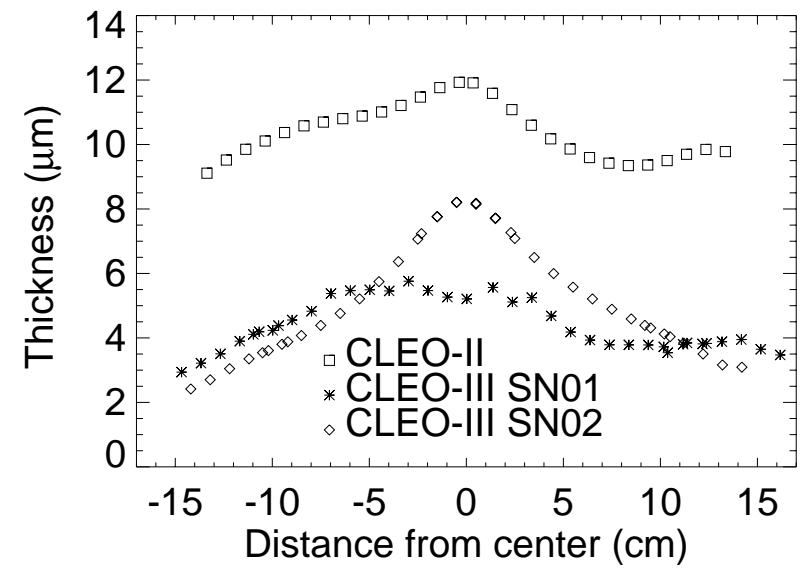

Figure 3: Gold thickness uniformity results for three Be beampipes measured by $x$-ray absorption.

film passed this test, it could not be removed by any others means. The principal function of the witness coupons which were coated with the beampipe was to subject them to these adhesion tests. No tests were performed on the Be beampipe itself in order to maintain UHV cleanliness.

The various factors which affect thickness uniformity described above were discovered in the development stage through repeated test coupon runs. The thickness was determined by weighing coupons before and after coating.

The thickness uniformity of the coated Be beampipes was measured by a simple $x$-ray absorption method performed at the Cornell High-Energy Synchrotron Source (CHESS). The attenuation of an $x$-ray beam with energy just below the gold $L_{I I I}$ edge was measured as a function of the longitudinal position on the beampipe. The thickness profiles for the three coated Be beampipes is shown in Figure 3. Uniformity is good for two pipes, but the third is thicker in the middle due to a non-uniform gold cathode, as described above. It should be mentioned that the detector tracking volume is within $\pm 6 \mathrm{~cm}$, so it is this thickness which is most important.

\section{REFERENCES}

[1] S. Henderson, Proc. Second Workshop on Backgrounds at the Machine Detector Interface, World Scientific (1998) 6.

[2] S. Henderson, Proc. 1997 Part. Acc. Conf., 291.

[3] S. Henderson, et. al., these proceedings.

[4] S. Henderson, Proc. 8th Mtg. DPF (1994) 1480.

[5] J.A. Thornton and A. S. Penfold, "Cylindrical Magnetron Sputtering”, in Thin Film Processes, ed. J.L. Vossen and W. Kern, Academic Press, New York, 1978.

[6] V. Malina et. al., Semi. Sci. Tech. 3 (1988) 1015.

[7] B.N. Chapman, J. Vac. Sci. Tech. 11 (1974) 106.

[8] P. Madakson, J. Appl. Phys. 70 (1991) 1380.

[9] M. Nayak et. al., J. Mat. Sci. Lett. 12 (1993) 119. 